${ }^{18}$ Malamy, M. H., Cold Spring Harbor Symp. Quant. Biol., 31, 189 (1966). ${ }^{19}$ Drake, J. W., J. Mol. Biol., 6, 268 (1963).

${ }_{20}$ Drake, J. W., and McGuire, J., Genetics, 55, 387 (1967).

${ }^{21}$ Tessman, I., Virology, 35, 330 (1968).

\section{Comparative Rates of Spontaneous Mutation}

RATES of spontaneous mutation suitable for making cornparisons between different species should consist of averages of rates at many sites, and should reflect all changes in DNA sequence in the tested regions. Average rates can be obtained by measurements of forward mutation, preferably within several different cistrons which should be totally dispensable for growth in permissive conditions, but sensitive even to missense mutations under restrictive or selective conditions. There are very few systems which fulfil most or all of these conditions (Table 1).

Table 1. COMPARATIVE Forward MUtation Rates

\begin{tabular}{lccc}
\multicolumn{1}{c}{ Organism } & $\begin{array}{c}\text { Base pairs } \\
\text { per genome }\end{array}$ & $\begin{array}{c}\text { Mutation rate } \\
\text { per base pair } \\
\text { replication }\end{array}$ & $\begin{array}{c}\text { Total } \\
\text { mutation } \\
\text { rate }\end{array}$ \\
Bacteriophage $\lambda$ & $4 \cdot 8 \times 10^{4}$ & $2 \cdot 4 \times 10^{-8}$ & $1.2 \times 10^{-3}$ \\
Bacteriophage T4 & $1.8 \times 10^{5}$ & $1 \cdot 7 \times 10^{-8}$ & $3.0 \times 10^{-8}$ \\
Salmonella typhimurium & $4 \cdot 5 \times 10^{6}$ & $2 \cdot 0 \times 10^{-10}$ & $0.9 \times 10^{-3}$ \\
Escherichia coli & $4 \cdot 5 \times 10^{8}$ & $2 \cdot 0 \times 10^{-10}$ & $0 \cdot 9 \times 10^{-3}$ \\
Neurospora crassa & $4 \cdot 5 \times 10^{7}$ & $0 \cdot 7 \times 10^{-11}$ & $2.9 \times 10^{-4}$ \\
Drosophila melanogaster & $2.0 \times 10^{8}$ & $7.0 \times 10^{-11}$ & 0.8 \\
Man & $2 \cdot 0 \times 10^{\circ}$ & $?$ & $?$
\end{tabular}

For all entries up to and including $N$. crassa, mutations arise predominantly during chromosomal replication ${ }^{1}$, and the mutation rate was calculated from the formula ${ }^{2}$

$$
m=0.4343\left(f-f_{0}\right) / \log \left(N / N_{0}\right)
$$

where $m$ is the mutation rate per chromosomal or nuelear replication, $f$ is the mutant frequency and $N$ is the population size. Where necessary, an average amino-acid was assumed to weigh 110 daltons, as calculated from average amino-acid frequencies among $E$. coli proteins ${ }^{3}$; and a gene was assumed to contain 1,000 base pairs, corresponding to a poly peptide weighing 36,700 daltons. The data for bacteriophage $\lambda$ are collected in ref. 4 ; the mutation rate was calculated for the $c$ I cistron, the protein of which weighs about 30,000 daltons. The data for bacteriophage T4 are collected in ref. 2 which are estimated to contain about 1,770 base pairs 5.6 . The bacterial dats are collected in refs. 2 and 7 ; the $E$. coli mutation rate was calculated for 5 ara cistrons, and the $S$. typhimurium mutation rate was calculated for 10 his cistrons. The mutation rate for the ad3 locus of $N$. crassa was calculated from the mutant frequency ${ }^{8}$ in a growth experiment encompassing about 60 generations (de Serres, personal communication; and ref. 9 ).

Mutations in Drosophila arise both during and independently of cell division ${ }^{10}$. An estimate of the total mutation rate per sexual generation per haploid set of chromosomes ${ }^{11}$ is $0 \cdot 4$, and an approximate mutation rate per base pair ${ }^{12}$ was calculated by assuming that all mutations arise during cel replication, and that an average of forty cell generations separate the fertilized egg from the progeny gametes. The human genome ${ }^{13}$ contains about 40 per cent of redundant species of nucleotide sequences ${ }^{14}$, the contribution of which to the mutational target size is unclear. Redundancy is not observed in the procaryotes, and is not yet reported in Drosophila.

Spontaneous mutation, expressed per base pair per replication, clearly proceeds at strikingly different rates in different organisms. This is also illustrated by the observation $^{4}$ that the temperate bacteriophage $\lambda$ assumes a mutation rate 20-100 times lower when multiplying as prophage (a set of bacterial genes) than when multiplying lytically (under autonomous control). Among the procaryotes, however, species differences in mutation rates largely disappear when rates are expressed per genome per replication : the mutation rate per base pair is inversely related to the size of the genome. Among the eucaryotes, very few data exist which can be transformed into average mutation rates, and it is not yet clear how these rates will vary with genome size.

If the constant mutation rate per genome suggested for procaryotes by Table $\mathbf{l}$ is general, then very small genomes should show very high mutation rates per cistron or per base pair. Comparisons should be sought in organisms which encode their own DNA polymerases and which are insulated from any but their own repair systems. Two likely candidates are Bacillus suhtilis bacteriophage $\varphi 29$ (ref. 15) and animal cell mitochondria ${ }^{16}$, the chromosomes of both of which are composed of double stranded DNA and are less than one tenth of the size of the chromosome of bacteriophage T4.

Observed mutation rates depend on several factors: the primary rate of damage or error insertion, the efficacy of repair, and the probability of detection of an altered phenotype. Decreased local mutation rates associated with increased genome size could be achieved by reducing the error rate, and also by increasing the sophistication of repair systems. (It is less clear whether mutations will tend to produce less drastically altered phenotypes in more complex organisms.) The spontaneous mutation rates listed in Table 1 are chiefly the result of point mutations, which may be divided into base pair substitutions and frameshift mutations. About 80 per cent of T4rII mutants contain frameshifts (although the leaky nature of many $r$ II base pair substitution mutants prevents their recovery) ${ }^{2}$. The frequencies of frameshift mutants are about $20-40$ per cent among S. typhimurium his mutants, about 15 per cent among $N$. crassa ad 3 mutants (where missense mutants are efficiently detected), and an indeterminant but significant percentage among $E$. coli lac mutants (see refs. in preceding article). Species specific differences in average mutation rates therefore reflect changes in both of the elementary types of point mutations. Because these clearly arise by a number of very different mechanisms ${ }^{2}$, alterations in average mutation rates must reflect changes in many enzymatic systems.

The frequent appearance of heritable changes in spontaneous mutation rates within a species emphasizes the extent to which mutation rates are genetically determined. Most of these heritable changes consist of mutator genes. In procaryotes, however, and particularly in those with small genomes, there exists the possibility of antimutator genes. These have now been described in bacteriophage T4 (preceding article).

The reasons for the approximately constant mutation rate per genome in the procaryotes are not yet clear. Mutation rates may be adjusted by natural selection to achieve a balance among the mostly deleterious effects of mutation, the need for variation, and the cost of suppressing mutation (for example, by slowing DNA synthesis, by introducing energy-consuming repair processes or by adopting informational redundancy). It can be imagined that procaryotic genomes are all so small that the intrinsic difficulties of achieving a sufficiently low total mutation rate are minor, and that more general factors (such as the ability to adapt rapidly versus the mutational load) are sufficient to fix the rate. At the other end of the scale, however, genome sizes may have expanded to the point where the cost of achieving the necessarily very small mutation rates per base pair becomes very large. It is therefore possible that the human total mutation rate is even larger than the very large Drosophila rate. Were this the case, the modern species, with its generally low numbers of offspring, might be particularly sensitive to increases in its mutation rate, such as may result from chemical modifications of the environment.

Department of Microbiology,

John W. Drake

University of Illinois,

Urbana, Illinois 61801 .

Received January 20, 1969.

1 Drake, J. W., Proc. US Nat. Acad. Sci., 55, 738 (1966).

2 Drake, J. W., An Introduction to the Molecular Basis of Mutation (HoldenDav, San Francisco, in the press).

${ }^{3}$ Sueoka, N., Proc. US Nat. Acad. Sci., 47, 1141 (1961).

$\checkmark$ Dove, W. F., Ann. Rev. Genetics, 2, 305 (1968).

${ }^{6}$ Stahl, F. W., Edgar, R. S., and Steinberg, J., Genetics, 50, 539 (1964). - Edgar, R. S, Feynman, R. P., Klein, S., Lielausis, I., and Steinberg, C. M.,
Genetics, $47,179(1962)$.

' Cairns, J., J. Mol. Biol., 6, 208 (1963).

${ }^{8}$ Brockman, H. E., and de Serres, F. J., Genetics, 48, 597 (1963).

- Horowitz, N. H., and MacLeod, H., Microbial Genetics Bull.,17, 6 (1960).

${ }^{10}$ Mullex, H. J., Prog. Nuclear Energy, Ser. VI, 2,146 (1959).

${ }^{11}$ Mukai, T., Genetics, 50, 1 (1964).

${ }^{12}$ Rudkin, G. T., Proc. XI Intern. Cong. Genet., 2, 359 (1963).

${ }^{19}$ Davidson, J. N., Leslie, I., and White, J. C., Lancet, i, 1287 (1951).

${ }^{14}$ Britten, R. J., and Kohne, D. E., Seience, 161, 529 (1968).

${ }^{15}$ Anderson, D. L., Hickman, D. D., and Reilly, B. E., J. Bacteriol., 81, $2081(1966)$.

${ }^{16}$ Meyer, R. R., and Simpson, M. V., Proc. US Nat. Acad. Sci., 61, 130 (1968). 